\title{
Yeni Bir İterasyon Yöntemi için Hemen-Hemen Büzülme Dönüşümleri Altında Bazı Sabit Nokta Teoremleri
}

\author{
Some Fixed Point Theorems for a New Iteration Method Under Almost Contraction \\ Mappings
}

\author{
Yunus ATALAN ${ }^{1}$ \\ ${ }^{1}$ Aksaray Üniversitesi, Matematik Bölümü, 68100, Aksaray, Türkiye
}

\begin{abstract}
$\ddot{O} \mathbf{z}$
$\mathrm{Bu}$ makalede (1) ile verilen iterasyon yönteminden daha sade olan yeni bir iterasyon yöntemi tanımlanmıştır. $\mathrm{Bu}$ iterasyon yönteminin hemen hemen büzülme dönüşümü şartını sağlayan iki operatörün ortak sabit noktasına yakınsak olduğu ispatlanmıştır. Ayrıca yeni iterasyon yönteminin (1) ile verilen iterasyon yönteminden daha hızlı olduğu gösterilmiştir ve bu sonucu destekleyen bir nümerik örnek verilmiştir. Son olarak, hemen hemen büzülme dönüşümü şartını sağlayan iki operatör için yeni tanımlanan iterasyon kullanılarak veri bağl1lığı sonucu elde edilmiştir.
\end{abstract}

Anahtar Kelimeler: Yeni iterasyon yöntemi, Hemen hemen büzülme dönüşümleri, Yakınsaklık hızı, Veri bağlılığı

\begin{abstract}
Throughout history, the emergence of scientific knowledge in real life has been associated with fields such as Physics, Chemistry, Biology, Medicine, Economics, Computer. Under these names, each field contains many abstract or practical problems to implement in itself, or as a result of the association with one of the others. Mathematical models of such problems are either an equation type or an equation system. The methods that can be used for solving the obtained equation systems can be listed as differential-integral equations or operator-functional equations.
\end{abstract}

Generally, in research on the existence of solutions of many problems which are belong to integral equations, differential equations, partial differential equations, dynamic programming, system analysis and fractal modeling, fixed point theory emerges as a useful method. This theory can also be applied to problems encountered in approach theory, game theory, mathematical economics and applied sciences.

The mathematical modeling of the real-life problem in historical development first began with Isaac Newton's idea of modeling the movements of planets with mechanical laws. In differential calculus found by Newton and Leibniz at the same time, the Euler equation for dynamic systems, the Lagrange equation for motion, the Fourier equation for heat diffusion, the Navier-Stokes equation for viscosity and the movement of fluids, the Maxwell equation for electromagnetic field and Schrodinger and Dirac equations for quantum mechanics were solved with the help of differential equations; thus, many scientific and technological developments were opened up. With this rapid development of the differential calculus, many equations could be solved in closed form. However, qualitative and quantitative details which belong to the problem, along with initial and boundary values that are important for some equations, have become apparent with the application of the iterative method developed by Picard for the solution of differential equations.

Sorumlu yazar/Corresponding Author: Yunus ATALAN, Tel: 0382 2882122, e-posta: yunusatalan@aksaray.edu.tr Gönderilme/Submitted: 03.04.2018 Düzenleme/Revised: 24.09.2018 Kabul/Accepted: 26.09.2018 
With the help of the iteration method used in the integral or differential equations to be solved, the limit of obtained sequence gives the solution of equation. For this reason, the iteration methods are gradually developed from several equations of the type $f(x)=x$ whose solutions are fixed points of the $f$ function.

However, the largest share in the placement of applications, which are outside of the basic differential and integral equations, into an abstract framework belongs to Stefan Banach. Banach Contraction Principle (BCP), which is proved by Banach in his doctoral thesis, from Hilbert spaces to metric spaces, has given a new direction to the study of the existence of fixed points in any space. BCP, which is very useful in solving differential and integral equations of different kinds, is also used as an effective tool to solve nonlinear problems. In addition to having a wide applicability, researchers have generalized $\mathrm{BCP}$ by putting new conditions on mapping or space.

With the process that started with Picard, fixed point iteration methods have attracted the attention of many researchers because they have wide application areas in science and they have come up to these days as a large working area. In this process, a number of iteration methods have been developed for certain classes of mappings to investigate their strong convergence, equivalence of convergence, rate of convergence and whether fixed points of these mappings are data dependent.

The equivalence of convergence between two iterations is expressed as follows:

When an iteration method for a given mapping converges to the fixed point of this mapping, does the other method converge to the same point? Based on this problem, many researchers have studied the equivalence of convergence of iteration methods for various classes of mappings, and a large literature has been created in this sense.

For two iterative methods that are equivalent in the sense of convergence, the knowledge of which method converges faster than the other is of great importance in applied mathematics. In this context, the rate of convergence of the iteration methods, which are in literature and newly defined, has been compared for the different classes of mappings by many researchers.

When constructing an iteration method, another mapping can be used that is close enough to the mapping chosen and is called the approach operator. Moving from this approach operator accepting that it has a different fixed point, the questions of how close the fixed point of the chosen mapping and fixed point of this approach operator to each other is and how the distance between them will be calculated, have revealed the concept of data dependence of these fixed points.

In this work, we show that the new iteration method, which is simpler than the iteration method (1), converges strongly to the common fixed point of two operators satisfying almost contraction condition. Also, we prove that the new iteration method is faster than the iteration method (1) and in order to show the validity of this result we give a numerical example. Finally, we obtain a data dependence result for two operators satisfying almost contraction mappings condition using new iteration method.

Key Words: New iteration method, Almost contraction mappings, Rate of convergence, Data dependence

\section{GíRiș}

Sabit nokta teorisi, matematik dışında fizik, kimya, biyoloji, mühendislik ve ekonomi gibi bilimin birçok dalında geniş bir uygulama sahasına sahip olduğundan pek çok araştırmacının dikkatini çeken ilginç bir çalışma alanıdır. Sabit nokta tanımını hatırlatacak olursak; $X$ boştan farklı bir küme ve $U: X \rightarrow X$ herhangi bir dönüşüm olsun. Eğer

$$
U q=q
$$

olacak şekilde bir $q \in X$ varsa bu $q$ noktasına $U$ dönüşümünün sabit noktası denir ve $U$ 'nun tüm sabit noktaları kümesi $F(U)$ şeklinde gösterilir.

Sabit nokta teorisinin temel amac1 $F(U) \neq \varnothing$ olabilmesi için $X$ kümesinin veya $U$ dönüşümünün üzerine konulacak uygun şartları belirlemektir. Bir dönüşümün sabit noktasının varlığı gösterildikten sonra bu noktaya ulaşmak için adına iterasyon denilen algoritmalar tanımlanmıştır. İlk olarak Picard [1]'ın 
tanımladığı iterasyonla başlayan süreçte günümüze kadar birçok iterasyon yöntemi tanımlanmış ve belirli dönüşüm sınıfları için bu yöntemlerin kuvvetli yakınsaklığı (norm yakınsama), yakınsaklıklarının denkliği, yakınsama hızları ve bu dönüşümlerin sabit noktalarının veri bağlı olup olmadığı araştırılmıştır (bkz. [2]-[16]).

2011 yılında S.H. Khan [17] üç adımlı bir iterasyonu şu şekilde tanımlamıştır:

$$
\left\{\begin{array}{c}
x_{n+1}=\left(1-b_{n}-c_{n}\right) x_{n}+b_{n} T y_{n}+c_{n} S z_{n} \\
y_{n}=\left(1-\alpha_{n}-\beta_{n}\right) x_{n}+\alpha_{n} T x_{n}+\beta_{n} S z_{n} \\
z_{n}=\left(1-a_{n}\right) x_{n}+a_{n} S x_{n} \\
(n \in \square),
\end{array}\right.
$$

burada $\left\{\alpha_{n}\right\}_{n=1}^{\infty},\left\{\beta_{n}\right\}_{n=1}^{\infty},\left\{a_{n}\right\}_{n=1}^{\infty},\left\{b_{n}\right\}_{n=1}^{\infty}$ ve $\left\{c_{n}\right\}_{n=1}^{\infty}$ dizileri [0,1] aralığındadır. Bu iterasyondan esinlenerek tanımladığımız yeni iki adımlı iterasyon yöntemi ise şu şekildedir:

$$
\left\{\begin{aligned}
x_{n+1}= & T y_{n} \\
y_{n}= & \left(1-\alpha_{n}-\beta_{n}\right) x_{n}+\alpha_{n} T x_{n}+\beta_{n} S x_{n} \\
& (n \in[),
\end{aligned}\right.
$$

burada $\left\{\alpha_{n}\right\}_{n=1}^{\infty}$ ve $\left\{\beta_{n}\right\}_{n=1}^{\infty}$ dizileri de $[0,1]$ aralığındadır.

Bu çalışmada (2) ile verilen iki adımlı iterasyon yönteminden elde edilen dizinin hemen hemen büzülme dönüşümü şartını sağlayan iki operatörün ortak sabit noktasına yakınsadığı ispatlanmıştır. Ayrıca bu yakınsamanın (1) ile verilen iterasyondan elde edilen dizinin yakınsamasına göre daha hızlı olduğu gösterilmiştir. Ayrıca bu sonucu desteklemek için bir örnek üzerinden grafik ve tablo verilmiştir. Son olarak hemen hemen büzülme dönüşümü şartını sağlayan iki operatör için (2) ile verilen iterasyon yöntemi kullanılarak veri bağl1lığı sonucu elde edilmiştir.

Şimdi temel sonuçlarımızı elde etmek için ihtiyacımız olan bazı ön bilgileri verelim:

Lemma 1.[18] $\left\{a_{n}\right\}_{n=0}^{\infty}$ ve $\left\{b_{n}\right\}_{n=0}^{\infty}$ aşağıdaki eşitsizliği sağlayan, negatif olmayan iki dizi olsun:

$$
a_{n+1} \leq\left(1-\mu_{n}\right) a_{n}+b_{n}
$$

Burada her $n \geq n_{0}$ için $\mu_{n} \in(0,1)$ ve $\sum_{n=1}^{\infty} \mu_{n}=\infty$ ayrica $n \rightarrow \infty$ için $\frac{b_{n}}{\mu_{n}} \rightarrow 0$. Bu durumda $\lim a_{n}=0$.

Lemma 2.[19] $\left\{a_{n}\right\}$ negatif olmayan bir dizi, her $n \in \square$ için $\mu_{n} \in(0,1), \sum_{n=1}^{\infty} \mu_{n}=\infty$ ve $\eta_{n} \geq 0$ olsun. Her $n \geq n_{0}$ için

$$
a_{n+1} \leq\left(1-\mu_{n}\right) a_{n}+\mu_{n} \eta_{n}
$$

eşitsizliğinin sağlanacağı şekilde bir $n_{0}$ sayısı mevcut olsun. Bu durumda

$$
0 \leq \limsup _{n \rightarrow \infty} a_{n} \leq \limsup _{n \rightarrow \infty} \eta_{n}
$$

eşitsizliği sağlanır.

2003 yılında, Berinde hemen-hemen büzülme dönüşümünü şu şekilde tanımladı:

Tanım 1. [20] $X$ bir tam metrik uzay ve $T: X \rightarrow X$ bir dönüşüm olsun. Eğer her $x, y \in X$ için

$$
d(T x, T y) \leq \delta d(x, y)+L d(y, T x)
$$

olacak şekilde bir $\delta \in(0,1)$ sabiti ve bir $L \geq 0$ mevcut ise, $T$ 'ye bir hemen-hemen büzülme dönüşümü denir.

Berinde tarafindan ortaya konulan aşağıdaki teorem, (3) ile verilen şarta oldukça benzer ek bir şartla bir hemen-hemen büzülme dönüşümünün sabit noktasını teklikle belirlemeyi mümkün kılmaktadır: 
Teorem 1. [20] $X$ bir tam metrik uzay ve $T: X \rightarrow X$ dönüşümü aşağıdaki şartı sağlayan bir hemenhemen büzülme dönüşümü olsun: Eğer her $x, y \in X$ için

$$
d(T x, T y) \leq \delta d(x, y)+L_{1} d(x, T x)
$$

olacak şekilde $\delta \in(0,1)$ sabiti ve $L_{1} \geq 0$ mevcutsa, $T$ bir tek sabit noktaya sahiptir.

(2) ile verdiğimiz iterasyon yöntemi iki operatörle inşa edildiğinden (4) ile verilen şartı normlu uzaylarda iki operatör için yeniden düzenlemek zorundayız. Bunun en iyi yolu, her iki operatörün de normlu bir uzayda (4) ile verilen şartı ayrı ayrı sağlamasıdır. Yani, $\delta \in(0,1)$ sabiti ve bir $L \geq 0$ sayısı için

$$
\|T x-T y\| \leq \delta\|x-y\|+L .\|x-T x\|
$$

ve

$$
\|S x-S y\| \leq \delta\|x-y\|+L .\|x-S x\|
$$

eşitsizlikleri geçerlidir.

Tanım 2. [10] $\left\{u_{n}\right\}_{n=1}^{\infty}$ ve $\left\{v_{n}\right\}_{n=1}^{\infty}$ aynı $p_{*}$ sabit noktasına yakınsayan iki dizi olsun. Eğer,

$$
\lim _{n \rightarrow \infty} \frac{d\left(u_{n}, p_{*}\right)}{d\left(v_{n}, p_{*}\right)}=0
$$

ise $\left\{u_{n}\right\}_{n=1}^{\infty}$ dizisi $p_{*}$ noktasına $\left\{v_{n}\right\}_{n=1}^{\infty}$ dizisinden daha hızlı yakınsar denir.

Tanım 3. [19] $T_{1}, T_{2}: C \rightarrow C$ iki operatör olsun. Her $x \in C$ ve sabit bir $\varepsilon>0$ için $\left\|T_{1} x-T_{2} x\right\| \leq \varepsilon$ ise $T_{2}$ 'ye $T_{1}$ 'in

yaklaşım operatörü adı verilir.

\section{TEMEL SONUÇLAR}

Teorem $2 C$ kümesi, $X$ Banach uzayının boştan farklı, kapalı ve konveks alt kümesi ve $x_{1} \in C$ olmak üzere $T, S: C \rightarrow C$ tanımlı (4) şartını sağlayan hemen-hemen büzülme dönüşümleri ve $F(T) \cap F(S) \neq \varnothing$ olsun. $\left\{x_{n}\right\}_{n=1}^{\infty}$ (2) ile verilen iterasyondan elde edilen bir dizi ve $\left\{\alpha_{n}\right\}_{n=1}^{\infty},\left\{\beta_{n}\right\}_{n=1}^{\infty}$ kontrol dizileri [0,1] aralığına ait olmak üzere $\sum_{n=1}^{\infty}\left(\alpha_{n}+\beta_{n}\right)=\infty$ şartı sağlansın. Bu durumda $\left\{x_{n}\right\}_{n=1}^{\infty}$ dizisi $T$ ve $S$ operatörlerinin teklikle belirli olan ortak $p_{*}$ sabit noktasına kuvvetli yakınsar.

İspat $p_{*}$ '’n $T$ ve $S$ operatörlerinin teklikle belirli olan ortak sabit noktası olduğu (4)'ten kolaylikla görülebilir. $n \rightarrow \infty$ için $x_{n} \rightarrow p_{*}$ olduğunu göstermemiz gerekmektedir. (2) ve (4)'ten

$$
\begin{aligned}
\left\|y_{n}-p_{*}\right\| & =\left\|\left(1-\alpha_{n}-\beta_{n}\right) x_{n}+\alpha_{n} T x_{n}+\beta_{n} S x_{n}-p_{*}\right\| \\
& \leq\left(1-\alpha_{n}-\beta_{n}\right)\left\|x_{n}-p_{*}\right\|+\alpha_{n}\left\|T x_{n}-p_{*}\right\|+\beta_{n}\left\|S x_{n}-p_{*}\right\| \\
& \leq\left(1-\alpha_{n}-\beta_{n}\right)\left\|x_{n}-p_{*}\right\|+\alpha_{n} \delta\left\|x_{n}-p_{*}\right\|+\beta_{n} \delta\left\|x_{n}-p_{*}\right\| \\
& =\left[1-\left(\alpha_{n}+\beta_{n}\right)(1-\delta)\right]\left\|x_{n}-p_{*}\right\|
\end{aligned}
$$

ve

$$
\left\|x_{n+1}-p_{*}\right\| \leq \delta\left\|y_{n}-p_{*}\right\|
$$

eşitsizlikleri elde edilir. (5) eşitsizliği (6)'da yerine yazılırsa, 


$$
\left\|x_{n+1}-p_{*}\right\| \leq \delta\left[1-\left(\alpha_{n}+\beta_{n}\right)(1-\delta)\right]\left\|x_{n}-p_{*}\right\|
$$

olacaktır. Son eşitsizlikte tümevarım uygulanırsa

$$
\begin{gathered}
\left\|x_{n}-p_{*}\right\| \leq \delta\left[1-\left(\alpha_{n-1}+\beta_{n-1}\right)(1-\delta)\right]\left\|x_{n-1}-p_{*}\right\| \\
\left\|x_{n-1}-p_{*}\right\| \leq \delta\left[1-\left(\alpha_{n-2}+\beta_{n-2}\right)(1-\delta)\right]\left\|x_{n-2}-p_{*}\right\| \\
\ldots \\
\left\|x_{1}-p_{*}\right\| \leq \delta\left[1-\left(\alpha_{1}+\beta_{1}\right)(1-\delta)\right]\left\|x_{0}-p_{*}\right\|
\end{gathered}
$$

olur. (7)'den

$$
\left\|x_{n+1}-p_{*}\right\| \leq\left\|x_{1}-p_{*}\right\| \delta^{n} \prod_{i=1}^{n}\left[1-\left(\alpha_{i}+\beta_{i}\right)(1-\delta)\right]
$$

yazılabilir.

$\delta \in(0,1)$ olduğundan $\left[1-\left(\alpha_{n}+\beta_{n}\right)(1-\delta)\right] \leq 1$ olur. Klasik analizden her $x \in[0,1]$ için $1-x \leq e^{-x}$ eşitsizliğinin sağlandığı bilinmektedir. Bu gerçekten hareketle (8) eşitsizliği

$$
\begin{aligned}
\left\|x_{n+1}-p_{*}\right\| & \leq\left\|x_{1}-p_{*}\right\| \delta^{n} \prod_{i=1}^{n} e^{-(1-\delta)\left(\alpha_{i}+\beta_{i}\right)} \\
& =\left\|x_{1}-p_{*}\right\| \delta^{n} e^{-(1-\delta) \sum_{i=1}^{n}\left(\alpha_{i}+\beta_{i}\right)}
\end{aligned}
$$

şeklinde yazılabilir. Son eşitsizlikte limit alınırsa $n \rightarrow \infty$ için $x_{n} \rightarrow p_{*}$ olduğu görülür. $\mathrm{Bu}$ ise ispatı tamamlar.

Teorem $3 C$ kümesi $X$ Banach uzayının boştan farklı, kapalı ve konveks alt kümesi olmak üzere $T, S: C \rightarrow C$ tanımlı (4) şartını sağlayan hemen-hemen büzülme dönüşümleri ve $F(T) \cap F(S) \neq \varnothing$ olsun. $u_{1}=x_{1} \in C$ olmak üzere, $\alpha_{n}>\mu>0$ veya $\beta_{n}>\mu>0$ şartları altında (2) ile verilen iterasyon yönteminden elde edilen $\left\{x_{n}\right\}_{n=1}^{\infty}$ dizisini ve $b_{n}+c_{n}<\frac{1}{1-\delta}$ ve $\lim _{n \rightarrow \infty}\left(b_{n}+c_{n}\right)=0$ şartları altında (1) iterasyon yönteminden elde edilen $\left\{u_{n}\right\}_{n=1}^{\infty}$ dizisini göz önüne alalım. Bu durumda $\left\{x_{n}\right\}_{n=1}^{\infty}$ dizisi $T$ ve $S$ operatörlerinin teklikle belirli olan ortak $p_{*}$ sabit noktasına $\left\{u_{n}\right\}_{n=1}^{\infty}$ dizisine göre daha hızlı yakınsar.

İspat (8)'den aşağıdaki eşitsizliği yazabiliriz.

$$
\left\|x_{n+1}-p_{*}\right\| \leq\left\|x_{1}-p_{*}\right\| \delta^{n} \prod_{i=1}^{n}\left[1-\left(\alpha_{i}+\beta_{i}\right)(1-\delta)\right]
$$

Ayrica [17] no'lu referanstan

$$
\left\|u_{n+1}-p_{*}\right\| \leq\left\|u_{1}-p_{*}\right\| \prod_{i=1}^{n}\left[1-\left(b_{i}+c_{i}\right)(1-\delta)\right]
$$

eşitsizliği elde edilir.

(9) ve (10) eşitsizliklerinden

yazılabilir.

$$
\frac{\left\|x_{n+1}-p_{*}\right\|}{\left\|u_{n+1}-p_{*}\right\|} \leq \frac{\left\|x_{1}-p_{*}\right\| \delta^{n} \prod_{i=1}^{n}\left[1-\left(\alpha_{i}+\beta_{i}\right)(1-\delta)\right]}{\left\|u_{1}-p_{*}\right\| \prod_{i=1}^{n}\left[1-\left(b_{i}+c_{i}\right)(1-\delta)\right]}
$$




$$
\psi_{n}=\frac{\delta^{n} \prod_{i=1}^{n}\left[1-\left(\alpha_{i}+\beta_{i}\right)(1-\delta)\right]}{\prod_{i=1}^{n}\left[1-\left(b_{i}+c_{i}\right)(1-\delta)\right]}
$$

şeklinde tanımlayalım. Bu durumda

olup hipotezden

$$
\frac{\psi_{n+1}}{\psi_{n}}=\frac{\delta\left[1-\left(\alpha_{n+1}+\beta_{n+1}\right)(1-\delta)\right]}{\left[1-\left(b_{n+1}+c_{n+1}\right)(1-\delta)\right]}
$$

i) $\alpha_{n}>\mu$ veya $\beta_{n}>\mu \Rightarrow \alpha_{n}+\beta_{n}>\mu$

$$
\begin{aligned}
& \Rightarrow(1-\delta)\left(\alpha_{n}+\beta_{n}\right)>\mu(1-\delta) \\
& \Rightarrow 1-(1-\delta)\left(\alpha_{n}+\beta_{n}\right)<1-\mu(1-\delta)
\end{aligned}
$$

ve

ii) $b_{n}+c_{n}<\frac{1}{1-\delta} \Rightarrow\left(b_{n}+c_{n}\right)(1-\delta)<1$

elde edilir. $\mathrm{O}$ halde

$$
\begin{aligned}
\frac{\psi_{n+1}}{\psi_{n}} & =\frac{\delta\left[1-\left(\alpha_{n+1}+\beta_{n+1}\right)(1-\delta)\right]}{\left[1-\left(b_{n+1}+c_{n+1}\right)(1-\delta)\right]} \\
& \leq \frac{\delta[1-\mu(1-\delta)]}{\left[1-\left(b_{n+1}+c_{n+1}\right)(1-\delta)\right]}
\end{aligned}
$$

olur. Ayrıca $\lim _{n \rightarrow \infty}\left(b_{n}+c_{n}\right)=0$ olduğundan

$$
\lim _{n \rightarrow \infty} \frac{\delta[1-\mu(1-\delta)]}{\left[1-\left(b_{n+1}+c_{n+1}\right)(1-\delta)\right]}=\delta[1-\mu(1-\delta)]<1
$$

elde edilir. Bu nedenle $\lim _{n \rightarrow \infty} \psi_{n}=0$ olur. Tanım 2'den $\left\{x_{n}\right\}_{n=1}^{\infty}$ dizisi, ortak $p_{*}$ sabit noktasına $\left\{u_{n}\right\}_{n=1}^{\infty}$ dizisine göre daha hızlı yakınsar.

Örnek $1 X=\square$ ve $C=[0,1]$ olmak üzere $T, S: C \rightarrow C$ operatörleri her $x \in C$ için sırasıyla $T(x)=x-1+\frac{1}{e^{x}}$ ve $S(x)=\frac{x}{1+x}$ şeklinde tanımlansın. $T$ ve $S$ 'nin ortak sabit noktasının $p_{*}=0$ olduğu açıktır. Başlangıç değeri $x_{1}=0,99$ olmak üzere kontrol dizilerini $\alpha_{n}=\beta_{n}=a_{n}=b_{n}=c_{n}=\frac{1}{9}$ şeklinde seçelim. MATLABR2015a programı kullanılarak elde edilen aşağıdaki tablo, (2) ile verilen iterasyon yönteminin, (1) ile verilen iterasyon yönteminden

daha hızlı olduğunu göstermektedir: 
Tablo 1. Yakınsaklık hızı karşılaştırılması

\begin{tabular}{|c|c|c|}
\hline İterasyon Adım Sayıs1 & Yeni İterasyon Yöntemi & (1) ile verilen iterasyon yöntemi \\
\hline 1 & 0,99000000000000 & 0,990000000000 \\
\hline 2 & 0,28631303118539 & 0,85540857025091 \\
\hline 3 & 0,02914867897021 & 0,73958412716685 \\
\hline 4 & 0,00033163252152 & 0,64002711558702 \\
\hline 5 & 0,00000004344287 & 0,48104624276882 \\
\hline 6 & 0,00000000000000 & $\vdots$ \\
\hline$\vdots$ & $\vdots$ & 0,00000000000001 \\
\hline 277 & 0,00000000000000 & 0,00000000000000 \\
\hline
\end{tabular}

Tablo 1 göstermektedir ki, yeni tanımladığımız iterasyon yöntemi 6. adımda sabit noktaya ulaşırken (1) ile verilen iterasyon yöntemi 278. adımda sabit noktaya ulaşmıştır. Aşağıda verilen grafik bu durumu göstermektedir:

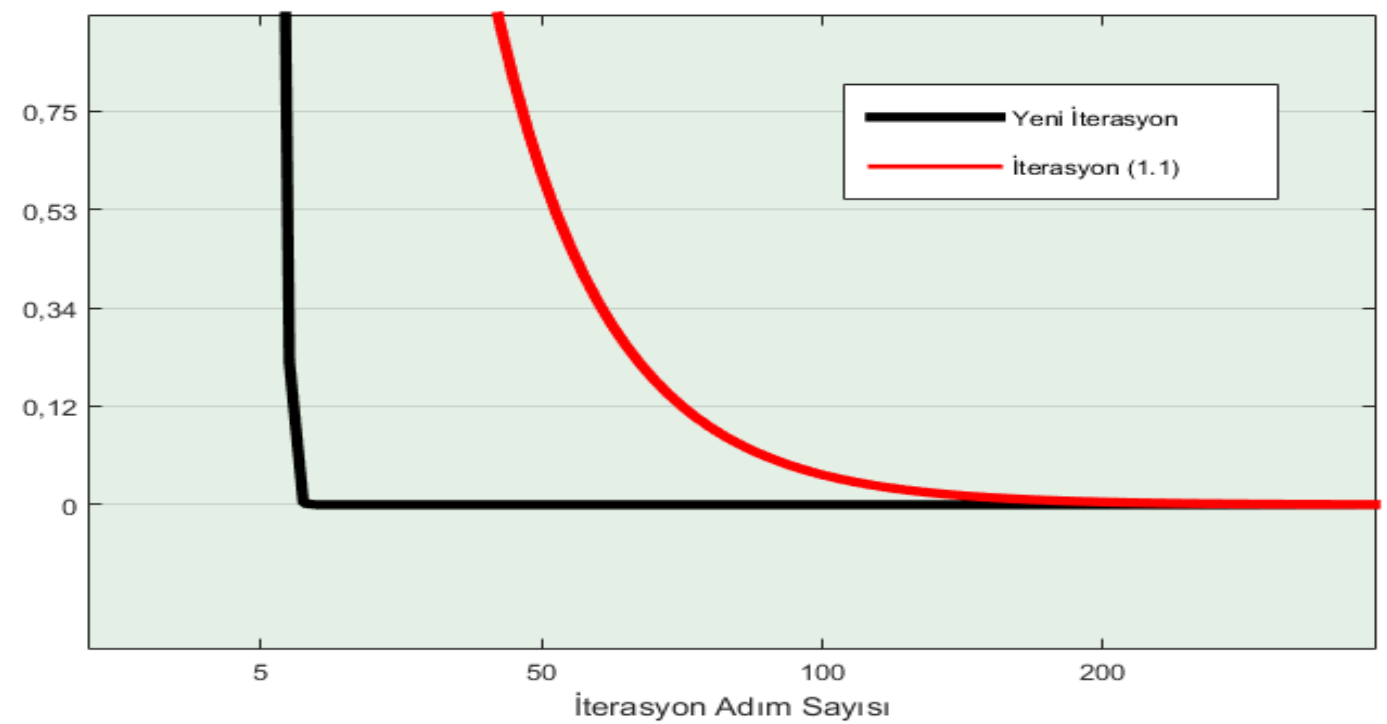

Şekil 1-İki iterasyon yöntemi için yakınsama hızlarının grafik gösterimi

Teorem $4 C$ kümesi, $X$ Banach uzayının boştan farklı, kapalı ve konveks alt kümesi olmak üzere $T, S: C \rightarrow C$ tanımlı (4) şartını sağlayan hemen-hemen büzülme dönüşümleri ve ortak sabit noktaları $p_{*}$ 
olsun. $T_{1}$ ve $S_{1}$ operatörleri ise $x_{*}$ ortak sabit noktasına sahip ve sırasıyla $T$ ve $S$ operatörlerinin yaklaşım operatörleri olsun. $T$ ve $S$ operatörleri kullanılarak inşa edilen (2) iterasyon yönteminden elde edilen dizi $\left\{x_{n}\right\}_{n=1}^{\infty}$ olmak üzere, $T_{1}$ ve $S_{1}$ operatörleri kullanılarak oluşturulan aşağıdaki iterasyon yönteminden elde edilen $\left\{u_{n}\right\}_{n=1}^{\infty}$ dizisini göz önüne alalım:

$$
\left\{\begin{array}{c}
u_{1} \in C, \\
u_{n+1}=T_{1} u_{n} \\
v_{n}=\left(1-\alpha_{n}-\beta_{n}\right) u_{n}+\alpha_{n} T_{1} u_{n}+\beta_{n} S_{1} u_{n}
\end{array}\right.
$$

Burada $\left\{\alpha_{n}\right\}_{n=1}^{\infty},\left\{\beta_{n}\right\}_{n=1}^{\infty}$ kontrol dizileri [0,1] aralığına ait olup her $n \in \square$ için $\frac{1}{2} \leq\left(\alpha_{n}+\beta_{n}\right)$ şartını sağlamaktadır. $T p_{*}=S p_{*}=p_{*}$ ve $T_{1} x_{*}=S_{1} x_{*}=x_{*}$ olmak üzere $n \rightarrow \infty$ için $u_{n} \rightarrow x_{*}$ ise, bu durumda $\varepsilon>0$ sayısı için

$$
\left\|p_{*}-x_{*}\right\| \leq \frac{3 \varepsilon}{1-\delta}
$$

eşitsizliği geçerlidir.

İspat (2), (4) ve (11) kullanılarak,

$$
\begin{aligned}
\left\|y_{n}-v_{n}\right\| \leq & \left(1-\alpha_{n}-\beta_{n}\right)\left\|x_{n}-u_{n}\right\| \\
& +\alpha_{n}\left\|T x_{n}-T_{1} u_{n}\right\|+\beta_{n}\left\|S x_{n}-S_{1} u_{n}\right\| \\
\leq & \left(1-\alpha_{n}-\beta_{n}\right)\left\|x_{n}-u_{n}\right\| \\
& +\alpha_{n}\left(\left\|T x_{n}-T u_{n}\right\|+\left\|T u_{n}-T_{1} u_{n}\right\|\right) \\
& +\beta_{n}\left(\left\|S x_{n}-S u_{n}\right\|+\left\|S u_{n}-S_{1} u_{n}\right\|\right) \\
\leq & {\left[1-\left(\alpha_{n}+\beta_{n}\right)(1-\delta)\right]\left\|x_{n}-u_{n}\right\| } \\
& +\left(\alpha_{n}+\beta_{n}\right) L\left\|x_{n}-T x_{n}\right\|+\left(\alpha_{n}+\beta_{n}\right) \varepsilon
\end{aligned}
$$

ve

$$
\begin{aligned}
\left\|x_{n+1}-u_{n+1}\right\| & =\left\|T y_{n}-T_{1} v_{n}\right\| \\
& \leq\left\|T y_{n}-T v_{n}\right\|+\left\|T v_{n}-T_{1} v_{n}\right\| \\
& \leq \delta\left\|y_{n}-v_{n}\right\|+L\left\|y_{n}-T y_{n}\right\|+\varepsilon
\end{aligned}
$$

elde edilir.

(12) eşitsizliği (13)’te yerine yazılırsa,

$$
\begin{aligned}
\left\|x_{n+1}-u_{n+1}\right\| \leq & \delta\left[1-\left(\alpha_{n}+\beta_{n}\right)(1-\delta)\right]\left\|x_{n}-u_{n}\right\| \\
& +\delta\left(\alpha_{n}+\beta_{n}\right) L\left\|x_{n}-T x_{n}\right\|+\delta\left(\alpha_{n}+\beta_{n}\right) \varepsilon \\
& +L\left\|y_{n}-T y_{n}\right\|+\varepsilon
\end{aligned}
$$

elde edilir. Hipotezden

$$
1-\left(\alpha_{n}+\beta_{n}\right) \leq\left(\alpha_{n}+\beta_{n}\right)
$$

olup $\delta \in(0,1)$ olduğundan (14) eşitsizliği aşağıdaki gibi yazılabilir:

$$
\begin{aligned}
\left\|x_{n+1}-u_{n+1}\right\| \leq & {\left[1-\left(\alpha_{n}+\beta_{n}\right)(1-\delta)\right]\left\|x_{n}-u_{n}\right\|+\left(\alpha_{n}+\beta_{n}\right) L\left\|x_{n}-T x_{n}\right\| } \\
& +\left(\alpha_{n}+\beta_{n}\right) \varepsilon+2\left(\alpha_{n}+\beta_{n}\right) L\left\|y_{n}-T y_{n}\right\|+2\left(\alpha_{n}+\beta_{n}\right) \varepsilon \\
= & {\left[1-\left(\alpha_{n}+\beta_{n}\right)(1-\delta)\right]\left\|x_{n}-u_{n}\right\| } \\
& +\left(\alpha_{n}+\beta_{n}\right)(1-\delta) \frac{\left\{L\left\|x_{n}-T x_{n}\right\|+2 L\left\|y_{n}-T y_{n}\right\|+3 \varepsilon\right\}}{(1-\delta)}
\end{aligned}
$$


Eğer

$$
\begin{aligned}
& a_{n}=\left\|x_{n}-u_{n}\right\| \\
& \mu_{n}=\left(\alpha_{n}+\beta_{n}\right)(1-\delta) \in(0,1) \\
& \eta_{n}=\frac{\left\{L\left\|x_{n}-T x_{n}\right\|+2 L\left\|y_{n}-T y_{n}\right\|+3 \varepsilon\right\}}{(1-\delta)}
\end{aligned}
$$

şeklinde seçilirse Lemma 2'den

$$
\begin{aligned}
0 & \leq \lim _{n \rightarrow \infty} \sup \left\|x_{n}-u_{n}\right\| \\
& \leq \lim _{n \rightarrow \infty} \sup \left\{\frac{\left\{L\left\|x_{n}-T x_{n}\right\|+2 L\left\|y_{n}-T y_{n}\right\|+3 \varepsilon\right\}}{(1-\delta)}\right\} \\
& =\frac{3 \varepsilon}{(1-\delta)}
\end{aligned}
$$

elde edilir. Teorem 2' den $x_{n} \rightarrow p_{*}$ elde edilmişti. Dolayısıyla

$$
\left\|p_{*}-x_{*}\right\| \leq \frac{3 \varepsilon}{1-\delta}
$$

elde edilir.

Teşekkür Yorum ve önerileriyle bu makaleye katkıda bulunan hakemlere teşekkür ederim.

\section{KAYNAKLAR}

[1] Picard, E., (1890). Mémoire Sur la Théorie des Équations Aux Dérivées Partielles et la Méthode des Approximations Successives. Journal de mathématiques pures et appliquées, 6, 145-210.

[2] Abbas, M., ve Nazir, T., (2014). A New Faster Iteration Process Applied to Constrained Minimization and Feasibility Problems. Matematıčkl Vesnıck, 66(2), 223-234.

[3] Agarwal, R., O Regan, D., ve Sahu, D., (2007). Iterative Construction of Fixed Points of Nearly Asymptotically Nonexpansive Mappings. Journal of Nonlinear and Convex Analysis, 8(1), 61-79.

[4] Chugh, R., Kumar, V., ve Kumar, S., (2012). Strong Convergence of a New Three Step Iterative

Scheme in Banach Spaces. American Journal of Computational Mathematics, 2, 345-357.

[5] Dogan, K., ve Karakaya, V., (2014). On the Convergence and Stability Results for a New General Iterative Process. The Scientific World Journal, 2014, 1-8.

[6] Gürsoy, F., Karakaya, V., ve Rhoades, B.E., (2013). Data dependence results of new multistep

and S-iterative schemes for contractive-like operators. Fixed Point Theory and Applications, 2013, 1-12.

[7] Karakaya, V., Atalan, Y., Dogan, K., ve Bouzara, NEH., (2017). Some Fixed Point Results for

a New Three Steps Iteration Process in Banach Spaces. Fixed Point Theory, 18(2), 625-640.

[8] Ishikawa, S., (1974). Fixed Point By a New Iteration Method. Proceedings of the American Mathematical Society, 44, 147-150.

[9] Karahan, I., ve Ozdemir, M., (2013). A General Iterative Method for Approximation of Fixed Points and Their Applications. Advances in Fixed Point Theory, 3(3), 510-526. 
[10] Phuengrattana, W., ve Suantai, S., (2012). Comparison of the Rate of Convergence of Various

Iterative Methods for the Class of Weak Contractions in Banach Spaces. Thai Journal of Mathematics, 11(1), 217-226.

[11] Karakaya, V., Atalan, Y., Dogan, K., ve Bouzara, NEH., (2016). Convergence Analysis for a New Faster Iteration Method. Istanbul Commerce University Journal of Science, 15(30), 3553.

[12] Mann, W.R., (1953). Mean Value Methods in Iteration. Proceedings of the American Mathematical Society, 4(3), 506-510.

[13] Noor, M.A., (2000). New Approximation Schemes for General Variational Inequalities. Journal of Mathematical Analysis and Applications, 251(1), 217-229.

[14] Osilike, M., (1995). Stability Results for Fixed Point Iteration Procedures. Journal of the Nigerian Mathematical Society, 14(15), 17-29.

[15] Pheungrattana, W., ve Suantai, S., (2011). On the Rate of Convergence of Mann, Ishikawa, Noor and SP Iterations for Continuous on an Arbitrary Interval. Journal of Computational and Applied Mathematics, 235, 3006-3014.

[16] Khan, S.H., (2013). A Picard-Mann Hybrid Iterative Process. Fixed Point Theory and Applications, 69, 1-10.

[17] Khan, S. H., (2011). Common fixed points of quasi-contractive type operators by a generalized iterative process. IAENG International Journal of Applied Mathematics, 41(3), 260-264.

[18] Weng, X., (1991). Fixed Point Iteration for Local Strictly Pseudo-Contractive Mapping. Proceedings of the American Mathematical Society, 113(3), 727-731.

[19] Şoltuz, S.M., ve Grosan, T., (2008). Data dependence for Ishikawa iteration when dealing with

contractive like operators. Fixed Point Theory and Applications, 2008, 1-7.

[20] Berinde, V., (2003). On The Approximation of Fixed Points of Weak Contractive Mappings. Carpathian J. Math., 19(1), 7-22. 